\title{
A1-L1 $1_{0}$ phase boundaries and anisotropy via multiple-order-parameter theory for an fcc alloy
}

\author{
G. B. TANOĞLU ${ }^{\dagger}$ \\ Izmir Institute of Technology, Department of Mathematics, \\ Gulbahce koyu, 35437, Urla, Izmir, Turkey \\ R. J. BRAUN ${ }^{*}$ \\ Department of Mathematical Sciences, University of Delaware, \\ Newark, DE 19716, USA \\ AND \\ J. W. CAHN ${ }^{\S}$ AND G. B. MCFADDEN ${ }^{\mathbb{I}}$ \\ National Institute of Standards and Technology, \\ Gaithersburg, MD 20899, USA
}

[Received 20 June 2002 and in revised form 3 January 2003]

\begin{abstract}
The dependence of thermodynamic properties of planar interphase boundaries (IPBs) and antiphase boundaries (APBs) in a binary alloy on an fcc lattice is studied as a function of their orientation. Using a recently developed diffuse interface model based on three non-conserved order parameters and the concentration, and a free energy density that gives a realistic phase diagram with one disordered phase (A1) and two ordered phases $\left(\mathrm{L}_{2}\right.$ and $\left.\mathrm{L}_{0}\right)$ such as occur in the $\mathrm{Cu}-\mathrm{Au}$ system, we are able to find IPBs and APBs between any pair of phases and domains, and for all orientations. The model includes bulk and gradient terms in a free energy functional, and assumes that there is no mismatch in the lattice parameters for the disordered and ordered phases. We catalog the appropriate boundary conditions for all IPBs and APBs. We then focus on the IPB between the disordered A1 phase and the $\mathrm{L} 1_{0}$ ordered phase. For this IPB we compute the numerical solution of the boundary value problem to find its interfacial energy, $\gamma$, as a function of orientation, temperature, and chemical potential (or composition). We determine the equilibrium shape for a precipitate of one phase within the other using the Cahn-Hoffman " $\xi$-vector" formalism. We find that the profile of the interface is determined only by one conserved and one non-conserved order parameter, which leads to a surface energy which, as a function of orientation, is "transversely isotropic" with respect to the tetragonal axis of the $\mathrm{L1}_{0}$ phase. We verify the model's consistency with the Gibbs adsorption equation.
\end{abstract}

\section{Introduction}

In this paper we study the interphase boundaries (IPBs) between coexisting phases and antiphase boundaries (APBs) between domains of variants of the same phase that occur during order-disorder transitions in an fcc-based binary alloy. We employ a free energy density that provides a mean-field

\footnotetext{
†Email: GamzeTanoglu@iyte.edu.tr

ॠ Corresponding author. Email: braun@math.udel.edu

§mail: John.Cahn@nist.gov

II Email: mcfadden@nist.gov
} 
description of the fcc disordered phase (A1, in Strukturbericht notation) and two ordered phases, both of which have wide ranges of composition away from stoichiometry: the $\mathrm{Cu}_{3} \mathrm{Au}$ phase, typified by the Strukturbericht $\mathrm{L}_{2}$ structure, and the $\mathrm{CuAu}(\mathrm{I})$ phase, typified by the Strukturbericht $\mathrm{L} 1_{0}$ structure, in the $\mathrm{Cu}-\mathrm{Au}$ system. The fcc lattice can be viewed as four interpenetrating simple cubic lattices (see Figure 1). In the disordered A1 structure, the four sublattices have equal probabilities of being occupied by either type of atom. In the $\mathrm{L}_{2}$ phase, one of the sublattices has a different occupation probability than the other three sublattices; for the $\mathrm{L} 1_{0}$ phase, two of the sublattices are occupied differently than the other two.

In previous work by the authors [5, 7, 6], a free energy density was employed which provided a useful description of $\mathrm{A} 1-\mathrm{L} 1_{2}$ IPBs and $\mathrm{L}_{2}$ APBs. In that model, however, the resulting phase diagram featured a multicritical point for all three phases [41], rather than the separate congruent disordering points with first order $\mathrm{A} 1-\mathrm{L} 1_{2}$ and $\mathrm{A} 1-\mathrm{L} 1_{0}$ transitions, as commonly observed in fcc systems such as $\mathrm{Cu}-\mathrm{Au}$. There was no co-existence of the $\mathrm{A} 1$ and $\mathrm{L} 1_{0}$ phases; the $\mathrm{A} 1-\mathrm{L} 1_{0}$ transition occurred only at the multicritical point and was second order. A more realistic phase diagram can be obtained with the improved free energy that we employ here (see Figure 2); there is $\mathrm{A} 1-\mathrm{L}_{2}$ and $\mathrm{A} 1-\mathrm{L} 1_{0}$ coexistence and the transitions at the congruent points are both first order [8]. We are then able to obtain $\mathrm{A} 1-\mathrm{L} 1_{0}$ IPBs, and verify the thermodynamic consistency of the resulting model by examining, for example, the relationship due to Gibbs between changes in $\gamma$ and the solute adsorption and excess entropy. We also compute the surface energy anisotropy of this IPB.

Recently, a number of continuum theories of phase change have used combinations of conserved and non-conserved order parameters with diffuse interfaces to predict or explain various phenomena in, for example, the solidification of binary alloys [54, 9, 55, 1, 4] and Ostwald ripening [16]. One method for treating diffuse interfaces is to use a free energy functional for the system based on continuum parameters that are spatially varying. The functional is written as the integral of the sum of two kinds of terms: bulk energies that are multiple-well functions of these parameters and gradient energies that are (generally quadratic) functions of the gradients of the order parameters. Both terms contribute to the energy in the transition regions that separate bulk phases; such gradient energy models date back more than a century [46, 52].

When there is a single non-conserved scalar order parameter, the usual form of the resulting equation is the Cahn-Allen equation [12,2]. When there is a single conserved parameter, say composition, the result is the Cahn-Hilliard equation [10,21] used to describe the spinodal decomposition of a binary alloy. Phase-field descriptions of the solidification of binary alloys combine elements of both Cahn-Allen and Cahn-Hilliard models (e.g., [54, 9, 55, 1, 4]).

Modeling that is based on a single composition variable and one order parameter cannot fully describe the ordering in binary fcc alloys, such as $\mathrm{Cu}-\mathrm{Au}$, which have three or more different crystal structures, and many possible interfaces, such as IPBs between different structures and APBs between domains or variants of the same ordered structures. The possible crystal structures include the disordered fcc phase and two ordered phases with the prototype $\mathrm{Cu}_{3} \mathrm{Au}$ and $\mathrm{CuAu}$ structures with Strukturbericht notations $\mathrm{A} 1, \mathrm{~L} 1_{2}$ and $\mathrm{L} 1_{0}$, respectively. The first mean-field calculation of a phase diagram for such a system [41] gave a multiphase critical point not seen in real phase diagrams and no coexistence between $\mathrm{A} 1$ and $\mathrm{L} 1_{0}$. A more sophisticated calculation using the cluster variation method (CVM) succeeded in obtaining a realistic phase diagram [32]. This method was used for calculating APBs in $\mathrm{L}_{2}$ and the $\mathrm{A} 1-\mathrm{L} 1_{2} \mathrm{IPB}$, but it is mathematically cumbersome and was used only for some low-index orientations [30]. By using three non-conserved order parameters and by adding appropriate higher-order terms to a mean-field free energy, it became possible to obtain 
realistic phase diagrams [8] with a free energy functional simple enough for interface property and profile calculations at all orientations [5, 7,6]; models in a similar vein have been developed by others [28, 18, 19, 34] and a more complete introduction is given in [7].

Single-order-parameter continuum formulations with a second order gradient energy term in cubic systems lead to isotropy of interfacial free energy $\gamma$, unless the anisotropy is introduced artificially with an orientation-dependent gradient coefficient [4, 33, 38, 50]. By anisotropy we mean how the properties of a planar interface depend on the orientation of its normal relative to the crystal axes. The multiple-order-parameter formulation leads to a natural anisotropy, i.e. without introducing ad hoc parameters in the model, in fcc [7] and even transverse to a six-fold axis in hcp [13]. Furthermore, the orientation dependence of interfacial properties (such as the interfacial energy and mobility [6]) is continuous and easily allows computation of the properties for all orientations.

In the modeling and computations by Braun et al. [7], the overall concentration was assumed to be uniform across the interface; the focus was on the role played by three non-conserved order parameters in determining the anisotropy of IPBs and APBs. The model was successful in giving the anisotropy of IPBs between the disordered A1 phase and the ordered $\mathrm{L}_{2}$ phase. The wetting transition of the APB was also described. But for an IPB, a uniform composition is inconsistent with the differences in the bulk concentrations in each phase at equilibrium as given by the phase diagram. Moreover, for both APBs and IPBs, the assumption of a uniform composition leads to no adsorption, so that finding a temperature and composition-dependent interfacial free energy leads to a violation of the Gibbs adsorption equation.

We report the methods and results detailed in a recent study which examined all these interfaces in a way that was consistent with the phase equilibria obtained with the improved free energy [48]. The results are calculations of equilibrium properties, including the interfacial free energy $\gamma$, and the determination of the interface profiles which describes how the order parameter and composition vary along the normal to the interface, for all of the various types of IPBs and APBs as a function of orientation, temperature and chemical potential (or composition). In this paper, we report the general methods for studying these interfaces, which includes a catalog of APBs and IPBs, and then focus on one specific example, the IPB between the disordered fcc phase (A1) and the ordered $\mathrm{L} 1_{0}$ phase. We allow the concentration to vary through the interface in a manner that is consistent with the phase diagram.

In this paper we do not consider elastic energy contributions. Our model implicitly assumes that there is no mismatch in the lattice parameters for the disordered and ordered phases, so that elastic effects can be neglected. In reality, there is always a change in the lattice parameters in an order-disorder transition, though the size of the mismatch can be small; in practice, commercial alloys often have additional chemical components added to produce lattice matching. In any event, the surface properties of IPBs naturally scale differently with length than the elastic effects, with surface effects dominating at small volumes. Here we effectively restrict attention to small length scales, for which the volumetric elastic contributions to the total energy are negligible compared to the surface energy contributions. The competition between surface energy and elastic energy has been studied by a number of authors (see, e.g., [24, 26, 27]). For example, Johnson and Cahn [24] studied a bifurcation in the shape of equilibrium particles that occurs with increasing particle size. For small particles the equilibrium shape is the Wulff shape entirely determined by minimization of surface energy. Beyond a critical size, elastic energy becomes a factor in the energy minimization. To minimize total energy these larger-sized particles resemble oblate ellipsoids, a compromise between the infinite plates that would minimize the elastic energy alone, and Wulff shapes that minimize the 
surface energy. In the present work we compute equilibrium shapes that resemble prolate (cigarshaped) ellipsoids entirely from minimizing surface energy for an $\mathrm{L} 1_{0}$ particle embedded in $\mathrm{A} 1$, or vice versa, in the absence of elastic effects. Muddle, Nie, and Hugo [39] have observed equilibrium $\mathrm{A} 1-\mathrm{L} 1_{0}$ microstructures in $\mathrm{Au}-\mathrm{Cu}$ systems with plate-like features that differ qualitatively from the equilibrium shapes that we compute. These larger-scale, plate-like structures have their origin in the theory of energy minimization in martensite transformations (see, e.g., [23]), which is beyond the scope of the present paper.

The present paper is organized as follows. The formulation is introduced in Section 2. We briefly explain the order parameters, the bulk free energy density, the bulk equilibrium states, the gradient energy contribution, and the catalog of all the interface types that can be studied with our model. The numerical method for the solution of the governing equations is presented in Section 3. In Section 4 some $\mathrm{A} 1-\mathrm{L} 1_{0}$ IPB results are discussed, including the verification of the Gibbs adsorption equation for an $\mathrm{A} 1-\mathrm{L} 1_{0}$ IPB and the transverse isotropy of the surface energy relative to the $c$-axis of the tetragonal $\mathrm{L}_{0}$ phase. The resulting anisotropic interface energies, their profiles, and the associated equilibrium shape for particles of one phase inside another are also given, based on Cahn-Hoffman $\xi$-vector formalism. Finally, a summary and discussion is given in Section 5.

\section{Formulation}

For a binary alloy, conventional continuum theories for equilibria and kinetic processes on lattices are based on the assumption that the site occupation densities are varying slowly compared to the atomic spacing. This assumption is not valid when there is ordering; the occupation densities of adjacent sites will vary greatly if the sites belong to different ordering sublattices. Instead, by defining an occupation density $\rho_{j}$ on each of the sublattices we obtain a set of quantities that are constant in each domain of an equilibrated ordered phase, and are slowly varying through the interfaces compared to the atomic spacing [42].

To describe the various ordered phases that we wish to consider for an $\mathcal{A}-\mathcal{B}$ binary system, we choose four sublattices as shown in Fig. 1, and denote by $\rho_{j}$ the occupation density on each sublattice. Each density represents the local average atomic fraction of species $\mathcal{A}$ on that sublattice; their specification is assumed to characterize the local state of the crystal at a given temperature, $\bar{T}$.

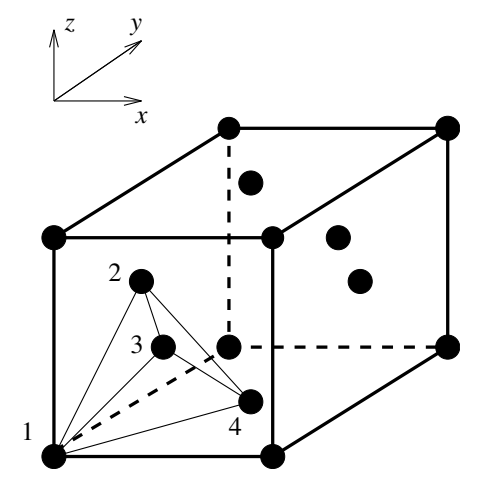

FIG. 1. A schematic diagram of an fcc lattice. There are four equivalent interpenetrating simple cubic sublattices. One sublattice corresponds to the corner of the conventional unit cell, and each of the other three corresponds to the center of a face intersecting at that corner. 


\subsection{The order parameters}

Since the differences in the $\rho_{j}$ describe the degree of order, it is convenient to introduce the parameters $W, X, Y$, and $Z$ defined by

$$
\begin{aligned}
W & =\frac{1}{4}\left(\rho_{1}+\rho_{2}+\rho_{3}+\rho_{4}\right), \\
X & =\frac{1}{4}\left(\rho_{1}+\rho_{2}-\rho_{3}-\rho_{4}\right), \\
Y & =\frac{1}{4}\left(\rho_{1}-\rho_{2}+\rho_{3}-\rho_{4}\right), \\
Z & =\frac{1}{4}\left(\rho_{1}-\rho_{2}-\rho_{3}+\rho_{4}\right) .
\end{aligned}
$$

This definition is consistent with a local representation

$$
\rho=W+X \cos (2 \pi x / a)+Y \cos (2 \pi y / a)+Z \cos (2 \pi z / a),
$$

where $x, y$, and $z$ range over the sublattices, and $a$ is the lattice parameter. The parameter $W$ thus represents the overall or mean atomic fraction of the system, and the coefficients $X, Y$, and $Z$ are non-conserved order parameters that can vary between $\pm 1 / 2$. In this model, the disordered A1 phase [17] is represented by $\rho_{1}=\rho_{2}=\rho_{3}=\rho_{4}=W$, which implies that $X=Y=Z=0$. The ordered $\mathrm{L} 1_{0}$ phase is described by pairs of sublattices with equal occupation densities, which leads to a single non-zero non-conserved order parameter, $X, Y$, or $Z \neq 0$. The ordered $\mathrm{L}_{2}$ phase is described by three sublattices whose densities are equal, and differ from the remaining sublattice density, which leads to non-conserved order parameters of equal (non-zero) magnitude, $X= \pm Y= \pm Z \neq 0$.

Scalar invariants of the order parameters are found from the symmetry of the fcc lattice [7, 34]. The first four are $X^{2}+Y^{2}+Z^{2}, X Y Z, X^{4}+Y^{4}+Z^{4}$, and $X^{2} Y^{2}+Y^{2} Z^{2}+Z^{2} X^{2}$.

\subsection{Bulk free energy density}

A thermodynamic description of a partially ordered crystal for the case of an isothermal system can be based on a Landau expansion of a generalized dimensionless scalar free energy density in terms of the scalar invariants [35],

$$
\begin{aligned}
\bar{F}(X, Y, Z, W, T)= & -\frac{\omega}{v_{m}}\left[e_{0}(W)+e_{2}(W)\left(X^{2}+Y^{2}+Z^{2}\right)+e_{3}(W) X Y Z+e_{41}\left(X^{4}+Y^{4}+Z^{4}\right)\right. \\
& \left.+e_{42}\left(X^{2} Y^{2}+X^{2} Z^{2}+Y^{2} Z^{2}\right)\right]+\frac{R \bar{T}}{4 v_{m}} \sum_{i=1}^{4} I\left(\rho_{i}\right)
\end{aligned}
$$

where the entropy term is taken as due to ideal mixing on each sublattice,

$$
I\left(\rho_{i}\right)=\rho_{i} \ln \left(\rho_{i}\right)+\left(1-\rho_{i}\right) \ln \left(1-\rho_{i}\right) .
$$

Here $\omega$ is the bond energy per mole, $R$ is the universal gas constant, $v_{m}$ is the molar volume; a bar denotes a dimensional variable. It is sometimes convenient to expand the entropy change on ordering in terms of these same invariants to eighth order. The ordering spinodal [20] for $\mathrm{L} 1_{0}$ occurs when $\partial^{2} F / \partial X^{2}=0$ at $X=Y=Z=0$. For $\mathrm{L} 1_{0}$ to form with a first order phase transition, it is necessary that $\partial^{4} F / \partial X^{4}<0 ; e_{41}$ has to be large and negative to overcome the positive entropy contribution.

If the internal energy $E$ is approximated by considering only pairwise bond energies with neighbors at any distance there will be only quadratic contributions to the energy and $e_{41}=0$. 
TABLE 1

Coefficients used for the internal energy contribution to the free energy density (after [8]); here $U=W-1 / 2$.

\begin{tabular}{lc}
\hline$e_{0}$ & $6 U^{2}$ \\
\hline$e_{2}$ & $-4+U^{2}$ \\
\hline$e_{3}$ & $200 U\left(1-2 U^{2}\right)$ \\
\hline$e_{41}$ & -12 \\
\hline$e_{42}$ & 15 \\
\hline
\end{tabular}

Multi-atom interactions among at least four neighbors must be considered to yield energy terms that are products of four or more $\rho$, which upon conversion to the $X, Y$, and $Z$ parameters give contributions to $e_{41}$. The coefficients used for the internal energy contribution to the free energy density that give the phase diagram in Fig. 2 are given in Table 1 . We will use these parameters for all computations in this paper.

\subsection{Bulk states}

For the free energy densities given in this section 2.3 , we have non-dimensionalized with $-\omega / v_{m}$ and the temperature has been non-dimensionalized with $-\omega / R$.

If the entropy is expanded to eighth order in the order parameters it is easy to show by minimizing $F$ with respect to the order parameters that the only minima in the free energy occur at $X=Y=Z=0$, at $|X|=|Y|=|Z| \neq 0$ with the signs chosen to make $e_{3} X Y Z<0$, and at $X, Y$, or $Z \neq 0$ with the other two order parameters equal to zero.

The trivial solution $X=Y=Z=0$ represents the disordered or A1 phase. For this phase, from (3) we have

$$
F_{\mathrm{A} 1}(W, T) \equiv F(0,0,0, W, T)=e_{0}(W)+T I(W) .
$$

For the $\mathrm{L} 1_{0}$ phase, corresponding to only one non-zero order parameter, the free energy is, again from (3),

$$
\begin{aligned}
F_{\mathrm{L} 1_{0}}(Z, W, T) \equiv F(0,0, Z, W, T)= & e_{0}(W)+e_{2}(W) Z^{2}+e_{41} Z^{4} \\
& +\frac{T}{2}[I(W+Z)+I(W-Z)] .
\end{aligned}
$$

Possible variants are $Z \neq X=Y=0, X \neq Y=Z=0$ or $Y \neq X=Z=0$. This phase corresponds to alternating layers of uniform occupation densities, which is the $\mathrm{CuAu}(\mathrm{I})$ phase in the $\mathrm{Cu}-\mathrm{Au}$ system [44]. Finally, for the $\mathrm{L} 1_{2}$ phase where $|X|=|Y|=|Z| \neq 0$, the free energy (3) becomes (with $e_{3}(W) Z^{3}<0$ )

$$
\begin{aligned}
F_{\mathrm{L1}_{2}}(Z, W, T) \equiv F(Z, Z, Z, W, T)= & e_{0}(W)+3 e_{2}(W) Z^{2} \\
& +e_{3}(W) Z^{3}+3\left(e_{41}+e_{42}\right) Z^{4} \\
& +\frac{T}{4}[I(W+3 Z)+3 I(W-Z)] .
\end{aligned}
$$


If $W \leqslant 1 / 2$ (see Table 1 ), $e_{3}$ is negative and the appropriate sign for the order parameter $Z$ is positive. When $W \geqslant 1 / 2, e_{3}$ is positive and $Z$ is negative. These two cases correspond to the two $\mathrm{L1}_{2}$ phases, e.g. $\mathrm{CuAu}_{3}$ and $\mathrm{Cu}_{3} \mathrm{Au}$.

Equilibrium phases can be found by convexification of the graphs of the three energies (5), (6), and (77) for fixed temperature. When there is a common tangent between the curves of two or three phases the points of tangency determine the compositions of the coexisting phases; the tangency points are found by solving non-linear algebraic equations. For example, for the coexistence of A1- $\mathrm{L} 1_{0}$ at temperature $T$, we must solve

$$
\begin{aligned}
& \frac{\partial F_{\mathrm{L} 1_{0}}}{\partial Z}\left(Z_{\mathrm{L} 1_{0}}, W_{\mathrm{L} 1_{0}}, T\right)=0, \\
& \frac{\partial F_{\mathrm{L} 1_{0}}}{\partial W}\left(Z_{\mathrm{L} 1_{0}}, W_{\mathrm{L}_{0}}, T\right)=\frac{\partial F_{\mathrm{A} 1}}{\partial W}\left(W_{\mathrm{A} 1}, T\right)=\mu, \\
& F_{\mathrm{A} 1}\left(W_{\mathrm{A} 1}, T\right)-F_{\mathrm{L} 1_{0}}\left(Z_{\mathrm{L} 1_{0}}, W_{\mathrm{L} 1_{0}}, T\right)-\mu\left(W_{\mathrm{L} 1_{0}}-W_{\mathrm{A} 1}\right)=0,
\end{aligned}
$$

for the unknowns $Z_{\mathrm{L} 1_{0}}, W_{\mathrm{L} 1_{0}}, W_{\mathrm{A} 1}$ and $\mu$ where the subscripts denote the values in that phase at coexistence. Similarly, we may solve the analogous non-linear systems of equations for the $A 1-\mathrm{L} 1_{2}$ and $\mathrm{L} 1_{2}-\mathrm{L} 1_{0}$ coexistences. These algebraic equations were all solved by using the software package DNSQ [47, 45].

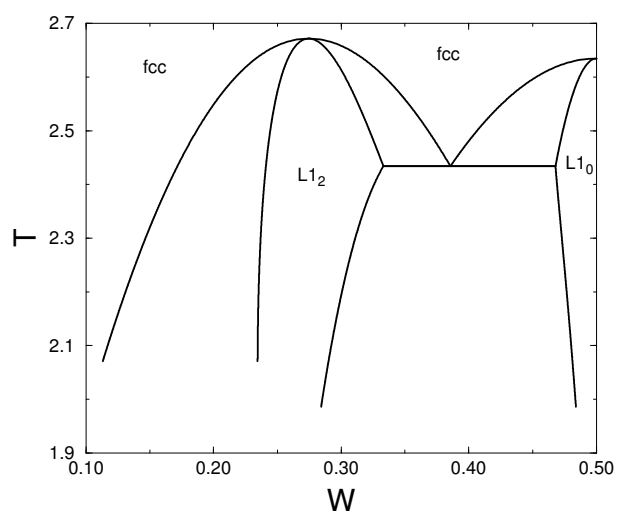

FIG. 2. A model phase diagram based on the $\mathrm{Cu}-\mathrm{Au}$ system, from the parameters in Table 1 note that it is symmetric about $W=1 / 2$. Here fcc denotes the disordered A1 phase.

Using the coefficients in Table 1 and plotting the tangent compositions as a function of temperature results in the equilibrium phase diagram of Fig. 2 (after Braun et al. [8]). We have chosen the coefficients so that the congruent temperature $T_{c}$ at $W=W_{c}=1 / 2$ occurs at $T=T_{c} \approx 2.635$. The congruent point is found from 88 with $W_{\mathrm{A} 1}=W_{\mathrm{L} 1_{0}}$; under these conditions, both phases have identical free energy densities as well. The phase diagram is an idealized version of the $\mathrm{Cu}-\mathrm{Au}$ phase diagram [44]. For example, our phase diagram is symmetrical about $W=1 / 2$ and does not have the orthorhombic $\mathrm{CuAu}$ (II) phase near $W=1 / 2$; $\mathrm{CuAu}$ (II) does not appear in most other theoretical phase diagrams, such as those from CVM calculations [17, 20, 31, 29].

The temperature of the congruent point for the $\mathrm{A} 1-\mathrm{L}_{2}$ transition can be normalized to a dimensional value of $\bar{T}_{c}=658 \mathrm{~K}$, appropriate for the Au-Cu system, by choosing the temperature 
scale $-\omega R / v_{m}=248 \mathrm{~K}$. This choice approximately fits the $\mathrm{Au}-\mathrm{Cu}$ phase diagram [44], but we have made no attempt beyond this to optimize the fit of the phase diagram to other experimentally measured data. Such a fit could certainly be done.

\subsection{The gradient energy}

The symmetries associated with the A1 crystal structure restrict the possible forms of gradient energy which must be added to the free energy density. By invoking the $F m \overline{3} m$ symmetry of the A1 crystal we find that the gradient energy term can be written in the relatively simple form [7, 34]

$$
\frac{A}{2}\left(X_{\bar{x}}^{2}+Y_{\bar{y}}^{2}+Z_{\bar{z}}^{2}\right)+\frac{B}{2}\left(X_{\bar{y}}^{2}+X_{\bar{z}}^{2}+Y_{\bar{x}}^{2}+Y_{\bar{z}}^{2}+Z_{\bar{x}}^{2}+Z_{\bar{y}}^{2}\right)+\frac{C}{2}|\bar{\nabla} W|^{2},
$$

where $A, B$, and $C$ are independent constants. Here subscripts denote partial derivatives, with $X_{\bar{x}}=$ $\partial X / \partial \bar{x}$, etc. The dimensional system free energy thus has the form ${ }^{*}$

$$
\begin{aligned}
\overline{\mathcal{F}}= & \int_{\bar{V}}\left\{\bar{F}(X, Y, Z, W, T)+\frac{A}{2}\left(X_{\bar{x}}^{2}+Y_{\bar{y}}^{2}+Z_{\bar{z}}^{2}\right)+\frac{B}{2}\left(X_{\bar{y}}^{2}+X_{\bar{z}}^{2}+Y_{\bar{x}}^{2}+Y_{\bar{z}}^{2}+Z_{\bar{x}}^{2}+Z_{\bar{y}}^{2}\right)\right. \\
& \left.+\frac{C}{2}|\bar{\nabla} W|^{2}\right\} \mathrm{d} \bar{V} .
\end{aligned}
$$

\subsection{Governing equations for interfaces}

We wish to find equilibria which connect two coexistence phases or two domains of an equilibrium along a planar boundary. To do this we minimize the functional

$$
\overline{\mathcal{I}}=\overline{\mathcal{F}}-\bar{\mu}_{0} \int_{\bar{V}} W \mathrm{~d} \bar{V}
$$

with respect to the non-conserved order parameters $X, Y, Z$ and the conserved order parameter $W$, with far-field boundary conditions imposed on the equilibrium phases. The second term in (11) represents a constraint on the amount of solute in the volume, and the Lagrange multiplier $\bar{\mu}_{0}$ will be seen to be the difference in the chemical potentials of the two species. When there is phase coexistence, $\bar{\mu}_{0}=\bar{\mu}$. Thus the governing equations are obtained as follows:

$$
\frac{\delta \overline{\mathcal{I}}}{\delta X}=\frac{\delta \overline{\mathcal{I}}}{\delta Y}=\frac{\delta \overline{\mathcal{I}}}{\delta Z}=\frac{\delta \overline{\mathcal{I}}}{\delta W}=0 .
$$

Evaluating the functional derivatives (see, e.g., [53]) gives

$$
\begin{aligned}
& 0=A X_{\bar{x} \bar{x}}+B X_{\bar{y} \bar{y}}+B X_{\bar{z} \bar{z}}-\bar{F}_{X}, \\
& 0=B Y_{\bar{x} \bar{x}}+A Y_{\bar{y} \bar{y}}+B Y_{\bar{z} \bar{z}}-\bar{F}_{Y}, \\
& 0=B Z_{\bar{x} \bar{x}}+B Z_{\bar{y} \bar{y}}+A Z_{\bar{z} \bar{z}}-\bar{F}_{Z}, \\
& 0=C\left(W_{\bar{x} \bar{x}}+W_{\bar{y} \bar{y}}+W_{\bar{z} \bar{z}}\right)-\bar{F}_{W}+\bar{\mu}_{0} .
\end{aligned}
$$

\footnotetext{
* Note the analogy with expressions for elastic energy in cubic crystals. If we let $(X, Y, Z)$ be the analog of displacement, $A$ is identified with $C_{11}$, B with $C_{44}$. The $C_{12}$ term is absent because of the fcc symmetry. We emphasize, however, that the non-conserved order parameters do not constitute the components of a tensor, nor do the gradient energy coefficients transform as a fourth-rank tensor, as discussed in Appendix B of [7][13].
} 
In this paper, we only consider stationary planar interfaces, and assume that the order parameters vary only in the direction parallel to the unit normal to the interface. We introduce a non-dimensional spatial variable, distance along the normal, defined as

$$
\zeta:=\frac{\hat{n} \cdot \overline{\mathbf{x}}}{L_{0}}
$$

where $\hat{n}=\left(n_{x}, n_{y}, n_{z}\right)$ denotes the unit normal, $\overline{\mathbf{x}}=(\bar{x}, \bar{y}, \bar{z})$ is the position vector and $L_{0}=$ $\sqrt{A v_{m} /(-\omega)}$ is the characteristic length scale. The temperature and the free energy density are made dimensionless with $-\omega / R$ and $-\omega / v_{m}$, respectively. This leads to the non-dimensional system of ordinary differential equations

$$
\begin{aligned}
\lambda_{x}^{2} X_{\zeta \zeta} & =F_{X}(X, Y, Z, W, T), \\
\lambda_{y}^{2} Y_{\zeta \zeta} & =F_{Y}(X, Y, Z, W, T), \\
\lambda_{z}^{2} Z_{\zeta \zeta} & =F_{Z}(X, Y, Z, W, T), \\
\delta^{2} W_{\zeta \zeta} & =F_{W}(X, Y, Z, W, T)-\mu_{0} .
\end{aligned}
$$

The coefficients in $(15)$ are given by

$$
\begin{aligned}
& \lambda_{x}^{2}=n_{x}^{2}+\epsilon^{2} n_{y}^{2}+\epsilon^{2} n_{z}^{2}, \\
& \lambda_{y}^{2}=\epsilon^{2} n_{x}^{2}+n_{y}^{2}+\epsilon^{2} n_{z}^{2}, \\
& \lambda_{z}^{2}=\epsilon^{2} n_{x}^{2}+\epsilon^{2} n_{y}^{2}+n_{z}^{2} .
\end{aligned}
$$

Here we have introduced the dimensionless parameters $\epsilon^{2}=B / A$ and $\delta^{2}=C / A$.

The right-hand sides in (15) are non-linear expressions which include polynomials up to fourth degree and logarithmic terms. Explicit expressions for the derivatives of $F$ are given in Appendix A.

The equations (15) govern the transitions between bulk states for any phase boundaries allowed in the phase diagram; the bulk states provide the appropriate far-field boundary conditions for the ordinary differential equations $(15)$ and together they constitute our model for IPBs.

\subsection{Catalog and symmetries of phase boundaries}

Only bulk state solutions representing the $\mathrm{A} 1, \mathrm{~L} 1_{2}$, and $\mathrm{L} 1_{0}$ phases occur in the phase diagram with $W<1 / 2$. In this section, we show that the symmetry of the fcc lattice and the free energy functional allow us to reduce the number of cases to three APBs and four IPBs, each for all orientations of $\hat{n}$. We note that the possibilities for APBs in this system have been enumerated by Mazauric [36], for example, but we wish to give a comprehensive list for both IPBs and APBs in the context of our model for convenience.

2.6.1 IPBs. For the $\mathrm{A} 1-\mathrm{L} 1_{2}$ interface the trivial solution $X=Y=Z=0$ represents the disordered A1 phase, while $X=Y=Z \neq 0$ represents the $\mathrm{L}_{2}$ phase. Although there are 4 different variants of this phase, they are equivalent by a translation by $\frac{a}{2}\langle 110\rangle$, where $a$ is the lattice parameter. Therefore only one possible set of boundary conditions needs to be considered for the A1- $1_{2}$ interface. The symmetry of the interfacial properties as a function of $\hat{n}$, such as $\gamma(\hat{n})$ and the Wulff shape, is $m \overline{3} m$. 
For the $\mathrm{A} 1-\mathrm{L} 1_{0}$ interface, we consider the case where $X=Y=0$ in both the bulk regions with $Z=Z_{\mathrm{L} 1_{0}} \neq 0$ in the bulk $\mathrm{L} 1_{0}$; i.e. making $z$ the four-fold or $c$-axis of $\mathrm{L} 1_{0}$. Since $F_{\mathrm{L} 1_{0}}$ and $Z_{\zeta}^{2}$ are even in $Z$, the resulting interfacial properties are identical for either sign of $Z_{\mathrm{L}_{0}}$. Similar symmetry arises for the other order parameters being non-zero, but the resulting energies are related by a rotation about $\langle 111\rangle$. Thus, we need only compute with a single set of boundary conditions for this case to observe the resulting interfaces and their properties. The symmetry of the interfacial properties is $4 / \mathrm{mmm}$.

For the $\mathrm{L}_{2}-\mathrm{L} 1_{0}$ interface, there are two possible boundary conditions for our system of equations. The order parameters may vary from $X=Y=Z=Z_{\mathrm{L}_{2}}>0$ (the $\mathrm{L}_{2}$ bulk state) to $X=Y=0$ and either to $Z=Z_{\mathrm{L} 1_{0}}>0$ or to $Z=-Z_{\mathrm{L} 1_{0}}$ (the $\mathrm{L}_{0}$ bulk state). All other possibilities are equivalent to one or the other of these cases. Therefore, there are two cases to be considered for $\mathrm{L} 1_{2}-\mathrm{L} 1_{0}$ interface. The symmetry of the interfacial properties is $4 / \mathrm{mmm}$ for both.

2.6.2 APBs. Antiphase boundaries separate two variants of the same ordered phase that necessarily share the same bulk free energy. For the $\mathrm{L}_{2}$ APB there are three possible displacement vectors that relate two variants: $\frac{a}{2}[011], \frac{a}{2}[101]$, and $\frac{a}{2}[110]$. The shifts along these vectors change the sign of two of the non-conserved order parameters. Only one four-fold axis is common to both domains because the shifts move the other two of the four-fold axes of one domain to coincide with two-fold axes of the other. The Wulff symmetry is $4 / \mathrm{mmm}$. Because of the symmetry of the free energy functional, all cases may be reduced to one possible set of boundary conditions; i.e., $X=Y=Z=Z_{\mathrm{L}_{2}}>0$ and $-X=-Y=Z=Z_{\mathrm{L}_{2}}>0$. All other combinations result in interfacial energies that are simply rotated and/or translated with respect to this case.

The $\mathrm{L}_{0}$ structure is formed by alternating planes of uniform occupation densities. Two possible sets of boundary conditions may be found by making changes between the two equal pairs of occupation densities. In the first set, three non-conserved order parameters may vary from $X=Y=0, Z=Z_{\mathrm{L} 1_{0}}$ to $X=Z=0, Y=Z_{\mathrm{L} 1_{0}}$, a 90 degree rotation of the layers. In the second set, they may vary from $X=Y=0, Z=Z_{\mathrm{L} 1_{0}}$ to $X=Y=0, Z=-Z_{\mathrm{L} 1_{0}}$. All other sets can be obtained from these two sets by using appropriate rotations, so only two sets of boundary conditions need to be considered for $\mathrm{L} 1_{0}$ APBs.

To summarize, there are a total of seven cases to be considered for IPBs and APBs in this model of the fcc binary alloy.

\section{Numerical methods}

To compute solutions of the non-linear system of ordinary differential equations (15) with the boundary conditions obtained from the phase diagram, we used two numerical methods. First, we used the boundary value problem solver COLNEW [3]; this package uses a Runge-Kutta basis on subintervals with variable spacing to provide the solution on an adaptive mesh. The solution is given as a list of coefficients for polynomials on the subintervals, but the solution may be accurately evaluated anywhere in the interval of the computation and so one is not limited to a given mesh.

In the second approach, we employed a second-order, centered finite difference approximation to the spatial derivatives and solved the resulting non-linear algebraic equations by using DNSQ [47, 45]. For the A1- $\mathrm{L} 1_{0}$ IPB, we can reduce the number of differential equations in [15] to two; this makes the second method reasonably efficient. Although the second method is slower than the first, the two methods are in good agreement (up to five significant digits) when both are used. 


\section{Results for the A1- $\mathrm{L1}_{0}$ IPB}

In the previous section we saw that the seven cases considered in [48] form a catalog. Although every case can be treated with the methods developed here, we consider here just one case to illustrate the methods: the IPBs connecting the disordered $\mathrm{A} 1$ phase and the ordered $\mathrm{L} 1_{0}$ phase near the upper right-hand corner of the phase diagram in Fig. 2, which could not be treated with the free energy used previously [7]. The remaining cases will be described separately [49].

For the $\mathrm{L}_{0}$ phase, $X=Y=0$ and only $Z$ and $W$ are non-zero. The bulk free energy density reduces to $F_{\mathrm{L} 1_{0}}\left(Z_{\mathrm{L}_{0}}, W_{\mathrm{L} 1_{0}}, T\right)$ as given in (6). We must solve (15) subject to the boundary conditions

$$
\begin{array}{r}
W=W_{\mathrm{A} 1}, X=Y=Z=0, \quad \text { as } \zeta \rightarrow-\infty, \\
W=W_{\mathrm{L} 1_{0}}, X=Y=0, Z=Z_{\mathrm{L}_{0}}, \quad \text { as } \zeta \rightarrow \infty .
\end{array}
$$

Numerically these conditions are applied at the ends of the computational domain, $z= \pm L$, and $L$ is made sufficiently large that the results are insensitive to it. The equations for $X$ and $Y$ in (15) have the solution $X(\zeta)=Y(\zeta)=0$, so we must solve the remaining equations for $Z$ and $W$.

When we have solutions for $Z$ and $W$, the interfacial energy $\gamma$ may be computed from either of the followings integrals:

$$
\gamma=\int_{-\infty}^{\infty}\left\{\epsilon^{2} Z_{\zeta}^{2}+\delta^{2} W_{\zeta}^{2}\right\} \mathrm{d} \zeta
$$

or

$$
\gamma=\int_{-\infty}^{\infty}\left\{\frac{\epsilon^{2}}{2} Z_{\zeta}^{2}+\frac{\delta^{2}}{2} W_{\zeta}^{2}+\Delta F\right\} \mathrm{d} \zeta
$$

The first integral of the Euler equations and boundary conditions yield

$$
\Delta F=F_{\mathrm{L}_{0}}(Z, W, T)-F_{\mathrm{L} 1_{0}}\left(0, W_{\mathrm{A} 1}, T\right)-\left(W-W_{\mathrm{A} 1}\right) F_{\mathrm{L}_{1}, W}\left(0, W_{\mathrm{A} 1}, T\right)=\frac{\epsilon^{2}}{2} Z_{\zeta}^{2}+\frac{\delta^{2}}{2} W_{\zeta}^{2} .
$$

Because of this equation the two expressions for $\gamma,(18)$ and (19), are equivalent.

The solution to the system of ordinary differential equations for the planar IPB (i.e. with $\zeta$ as the only independent variable) with the given boundary conditions with $X=Y=0$ is conjectured to give the unique minimum energy; if we have a unique minimum the appearance of additional phases in the interfacial region ("wetting behavior," see, e.g., [7]) does not occur for any orientation and temperature. Any proof that this solution to the ODE is a minimum applies only to the planar boundary and does not preclude the possibility of solutions having a microstructure with more complicated spatial dependence on $x, y$, and $z$ (e.g. a zig-zag boundary) that satisfy the system of partial differential equations (13) rather than the ordinary differential equations.

\subsection{Transverse isotropy of the surface energy}

The only dependence on the direction of the interface normal in the ordinary differential equations for $Z$ and $W$ is contained in $\lambda_{z}^{2}$. By expressing the components of the unit normal vector in spherical 
coordinates,

$$
n_{x}=\sin \phi \cos \theta, \quad n_{y}=\sin \phi \sin \theta, \quad n_{z}=\cos \phi,
$$

where $\phi$ is the polar angle and $\theta$ is the azimuthal angle, from 16 we find

$$
\lambda_{z}^{2}=\epsilon^{2} \sin ^{2} \phi+\cos ^{2} \phi=1+\left(\epsilon^{2}-1\right) \sin ^{2} \phi,
$$

which depends only on the polar angle, $\phi$. A function of the orientation that is independent of $\theta$, the azimuthal angle, is called transversely isotropic. We predict that $\gamma$ for the A1-L1 0 IPB should be transversely isotropic with respect to the $c$-axis of the tetragonal $\mathrm{L} 1_{0}$ phase.

Moreover, this equation relates $\phi$ and $\epsilon$ to a single computational variable, $\lambda_{z}$. Properties are not determined by $\phi$ and $\epsilon$, but by the value of $\lambda_{z}$ according to 22 . Computing interface properties for a range of $\lambda_{z}$ can be accomplished in a variety of ways. We have chosen to present interface profiles at $\phi=0$, i.e., for the [001] orientation with $\lambda_{z}^{2}=1$, and at $\phi=90^{\circ}$, i.e., for the [100] orientation with $\lambda_{z}^{2}=\epsilon^{2}$. Because of the transverse isotropy, we can use [100] to designate $\langle h k 0\rangle$ results, as well as $\left\langle h^{\prime} 0 l^{\prime}\right\rangle$ to designate $\langle h k l\rangle$ results, with $h^{\prime} / l^{\prime}=\sqrt{h^{2}+k^{2}} / l$. The orientation dependence of interfacial energy is computed for given values of $\epsilon^{2}$.

\subsection{The Gibbs adsorption equation}

We next verify that the appropriate form of the Gibbs adsorption equation holds for our model. This equation relates the variation, $\mathrm{d} \gamma / \mathrm{d} T$, of the surface energy of the IPB along the coexistence region of the phase diagram to the adsorption of solute and entropy at the interface (see, e.g., [37]). Following the development given by Cahn in [11], in Appendix B we show that this equation takes the form

$$
\frac{\mathrm{d} \gamma}{\mathrm{d} T}=-\int_{-\infty}^{\infty}\left\{\left(S-S_{\mathrm{A} 1}\right)-\left(W-W_{\mathrm{A} 1}\right) \frac{\left(S_{\mathrm{L} 1_{0}}-S_{\mathrm{A} 1}\right)}{\left(W_{\mathrm{L} 1_{0}}-W_{\mathrm{A} 1}\right)}\right\} \mathrm{d} \zeta,
$$

where $S=-\partial F / \partial T$ is the entropy density and $S_{\mathrm{A} 1}$ is its value in the bulk A1 phase, etc. We have computed the quantity $\mathrm{d} \gamma / \mathrm{d} T$ along the coexistence region and found that it agrees with our

\section{TABLE 2}

Comparison of $\mathrm{d} \gamma / \mathrm{d} T$ for $\mathrm{A} 1-\mathrm{L} 1_{0} I P B$ for several temperatures for $\epsilon^{2}=0.05, \delta^{2}=1.0$. For columns labeled "Eqn (23)," the composite trapezoidal rule was used to evaluate the integrals with numerically computed interface profiles. The columns labeled " $\mathrm{d} \gamma / \mathrm{d} T$ " used a centered finite difference approximation to the curve in the $(T, \gamma)$ plane. The results agree to within the error of the numerical methods used.

\begin{tabular}{ccccc}
\hline \multicolumn{2}{c}{$[001]$} & \multicolumn{2}{c}{$[100]$} \\
\hline$T$ & Eqn $[23)$ & $\mathrm{d} \gamma / \mathrm{d} T$ & Eqn $[23)$ & $\mathrm{d} \gamma / \mathrm{d} T$ \\
\hline 2.50 & 0.03174 & 0.03191 & -0.01660 & -0.01605 \\
\hline 2.55 & 0.03139 & 0.03139 & -0.01756 & -0.01755 \\
\hline 2.60 & 0.03099 & 0.03082 & -0.01870 & -0.01919 \\
\hline 2.62 & 0.03064 & 0.03059 & -0.01972 & -0.01989 \\
\hline 2.63 & 0.03045 & 0.03047 & -0.02015 & -0.02024 \\
\hline
\end{tabular}




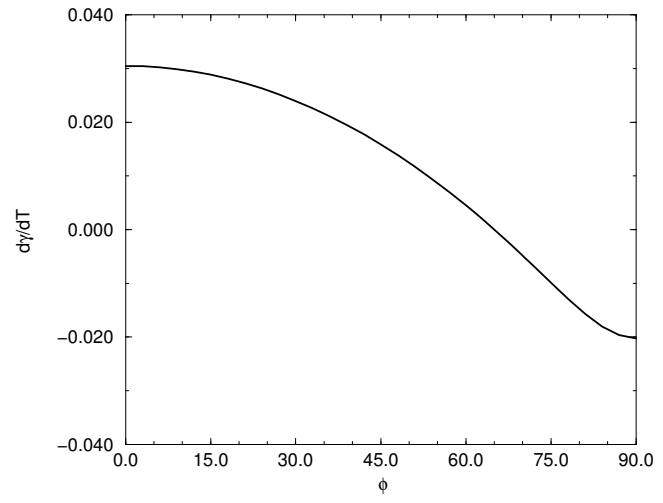

FIG. 3. $\mathrm{d} \gamma / \mathrm{d} T$ as a function of the azimuthal angle $\phi$ at $T=2.63$ for $\epsilon^{2}=0.05$ and $\delta^{2}=1.0$.

computed results for $\gamma(T)$ that were computed directly from the IPB profiles; results are given in Table 2 The first column is the temperature; column 2 is the result of evaluating (23) using the composite trapezoidal rule with computed data for the order parameters. Column 3 is a centered finite difference approximation to the numerical data plotted in Figure 9 for [001]. Columns 4 and 5 give analogous results for [100]. Good agreement is seen between the slope of the $\gamma(T)$ plot from both sources, verifying explicitly that our model and numerical solutions are consistent with the Gibbs adsorption equation.

The sign of $\mathrm{d} \gamma / \mathrm{d} T$ changes at orientations between [001] and [100]. Figure 3 shows $\mathrm{d} \gamma / \mathrm{d} T$ as a function of the azimuthal angle $\phi$; the zero of $\mathrm{d} \gamma / \mathrm{d} T$ occurs at about $65^{\circ}$.

\subsection{Interface profile}

We next consider the interface profile and surface energy anisotropy at the congruent temperature, $T_{c} \approx 2.635$, and composition $W=1 / 2$, for representative orientations with $\epsilon^{2}=0.005$. Figures 4 and 5 show the interface profile for the [100] and [001] orientations, respectively, at the critical temperature. From 22) and the text after it, we can see that the [001] interface profile is independent of $\epsilon^{2}$. Also, the [100] orientation has a sharper interface than the [001] orientation for decreasing

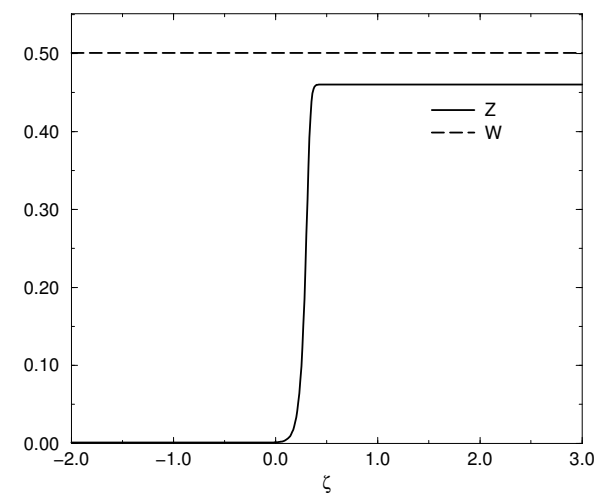

FIG. 4. A1-L $1_{0}$ IPB for an $\langle h k 0\rangle$ orientation. Here $\delta^{2}=1.0, \epsilon^{2}=\lambda_{z}^{2}=0.005$, and $T=T_{c}$. 


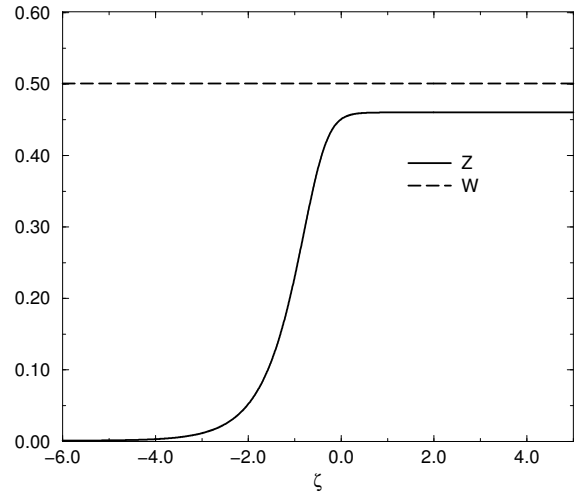

FIG. 5. A1-L $1_{0} \mathrm{IPB}$ for the [001] orientation. Here $T=T_{c}, \delta^{2}=1.0$, and $\epsilon^{2}$ is arbitrary.

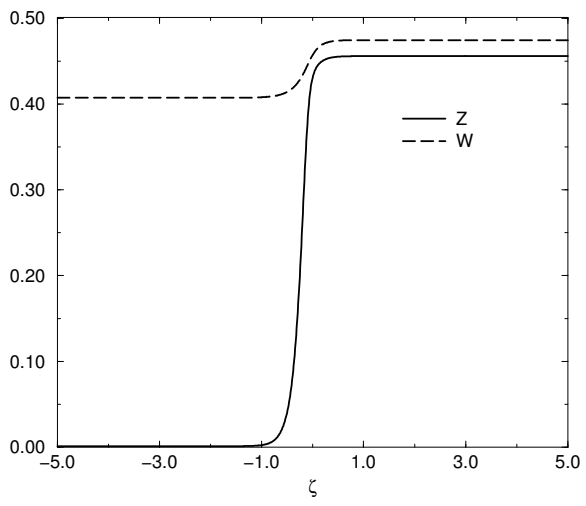

FIG. 6. A1-L1 $1_{0}$ IPB for the [100] orientation. Here $\delta^{2}=1.0, \epsilon^{2}=\lambda_{z}^{2}=0.005$, and $T=2.5$.

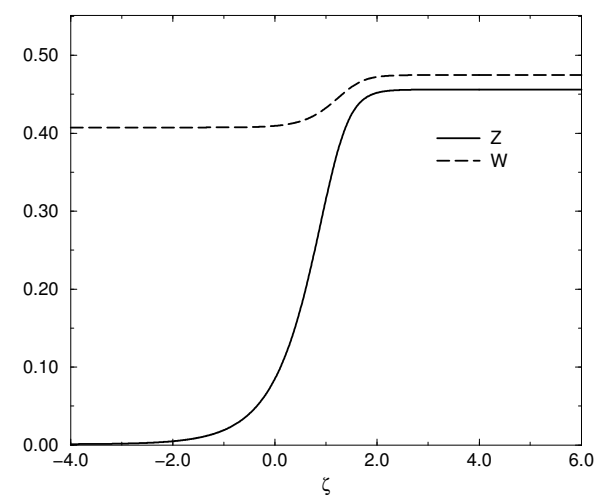

FIG. 7. A $1-\mathrm{L} 1_{0}$ IPB for the [001] orientation. Here $T=2.5, \delta^{2}=1.0$, and $\epsilon^{2}$ is arbitrary. 
$\epsilon^{2}<1$. The phase diagram shown in Fig. 2 is symmetric about $W=1 / 2$; therefore $W$ has the same value through the interfacial region and bulk states.

We next consider the case $T=2.5$ for [100] and [001] orientations. The interface profiles for these orientations are in Figs. 6 and 7 $Z$ is wider than $W$ for [001] in Fig. 7 It is again observed that the thickness of the interface for [100] is smaller than that of the interface for [001].

\subsection{Surface energy anisotropy}

Since $\gamma(\phi, \theta)=\gamma(\phi)$ we can represent $\gamma$ as a two-dimensional polar plot as shown in Fig. 8. As can be seen in this figure, the minimum energy occurs in the [100] direction and the maximum energy occurs in the [001] direction.

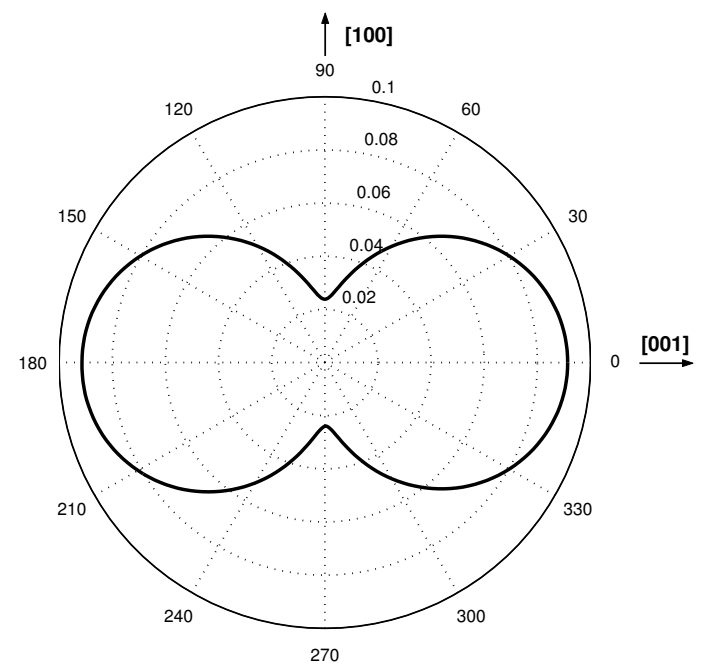

FIG. 8. $\gamma$-plot for $\mathrm{A} 1-\mathrm{L} 1_{0}$ IPB for $\langle h k 0\rangle$. Here $T=2.5, \delta^{2}=1.0, \epsilon^{2}=0.05$. Polar angle values are specified around the perimeter.

In Fig. 9 we compare the surface energies for [001] and [100] orientations as a function of temperature. The difference between the maximum and minimum energy becomes larger as the critical temperature $T_{c}$ is approached; i.e., the degree of anisotropy reaches a maximum value at $T_{c}$. Strong tetragonal anisotropy is found for the range of temperatures given in Fig. 10 for $\epsilon^{2}=0.05$. In CVM computation of interfacial properties for bcc [15] and fcc [30] materials, the difference between the interfacial energies increases at lower temperatures and then decreases at high temperatures. While it is common for materials to have the anisotropy decrease with increasing (high) temperature, it does not happen to be so for our model as we discuss shortly.

This trend of increasing anisotropy with temperature is amplified as $\epsilon$ decreases from unity. Numerical approximations of the interfacial energies at various orientations, for temperatures $T=T_{c}$ and $T=2.5$ and for $\epsilon^{2}=0.005$, are compared in Table 3 . By comparing Fig. 10 and Table 3, we observe that the level of anisotropy is increasing for smaller gradient energy coefficients $\left(\epsilon^{2}=0.005\right)$. 


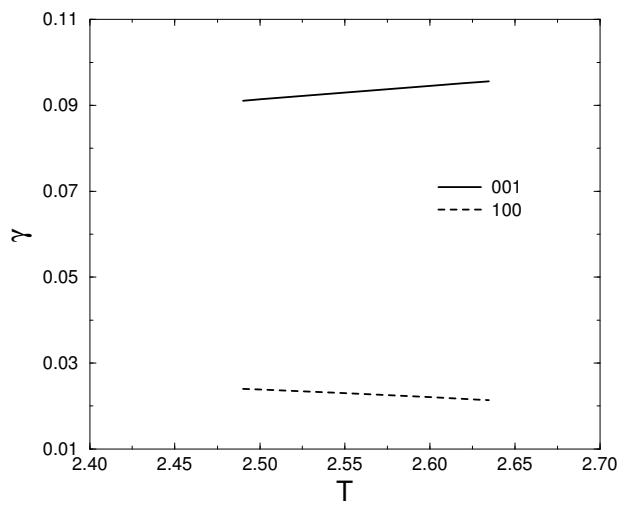

FIG. 9. Variation of interfacial energy with temperature for [001] and [100] orientations for A1-L $1_{0}$ IPB. Here $\delta^{2}=1.0$, $\epsilon^{2}=0.05$

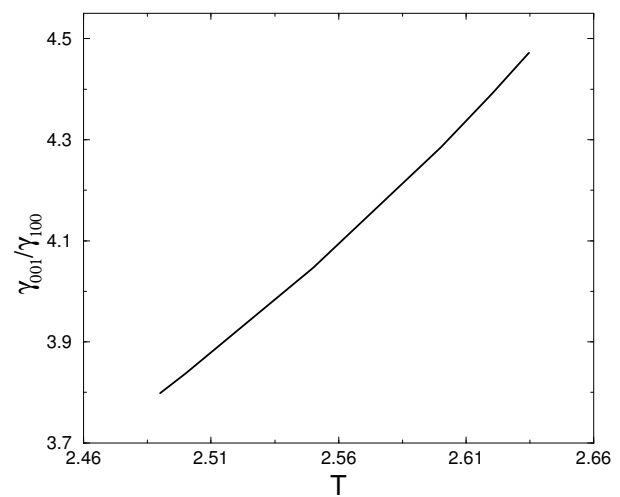

FIG. 10. Level of anisotropy for A1-L1 $1_{0}$ IPB given by $\gamma_{001} / \gamma_{100}$ versus temperature. Here $\delta^{2}=1.0, \epsilon^{2}=0.05$.

TABLE 3

Comparison of interfacial energy for $\mathrm{A} 1-\mathrm{L} 1_{0} I P B$ at $T=T_{c}$ and $T=2.5$ for $\epsilon^{2}=0.005, \delta^{2}=1.0$.

\begin{tabular}{ccc}
\hline & \multicolumn{2}{c}{$\gamma$} \\
\cline { 2 - 3 } orientation & $T=T_{c}$ & $T=2.5$ \\
\hline$[001]$ & 0.09559 & 0.09193 \\
{$[101]$} & 0.06776 & 0.06563 \\
{$[111]$} & 0.05546 & 0.05435 \\
{$[100]$} & 0.00676 & 0.01485 \\
\hline
\end{tabular}

In fact, the level of anisotropy increases dramatically as $\epsilon \rightarrow 0$ at the congruent point $(W, T)=$ $\left(1 / 2, T_{c}\right)$ because $\gamma_{100} \propto \epsilon$ there. This can be seen as follows. At the congruent point, the IPB profile satisfies $W=1 / 2$ and

$$
\lambda_{z}^{2} Z_{\zeta \zeta}=F_{z}\left(0,0, Z, 1 / 2, T_{c}\right)
$$


where $\lambda_{z}$ is given in (16). The interfacial energy $\gamma\left(n_{x}, n_{y}, n_{z}\right)$ computed from (24) then satisfies

$$
\frac{\gamma\left(n_{x}, n_{y}, n_{z}\right)}{\gamma_{001}}=\sqrt{\epsilon^{2}\left(n_{x}^{2}+n_{y}^{2}\right)+n_{z}^{2}}
$$

[cf. Equation (6.1) in [7] for the analogous APB result]. The ratio $\gamma_{001} / \gamma_{100}=\epsilon^{-1}$ therefore diverges in the limit as $\epsilon \rightarrow 0$ at the congruent point. When $\epsilon=1$, the IPBs are isotropic. In the model we must therefore see an increase in anisotropy as the congruent point is approached for small $\epsilon$, since the level of anisotropy away from the congruent point remains bounded due to the contribution from the variation of the concentration $W$ through the interface. We note that the limit $\epsilon \rightarrow 0$ corresponds to neglecting the second-nearest-neighbor interactions in the derivation of the gradient energy terms given in [7]; the remaining contributions from the nearest-neighbor terms are highly orientation-dependent.

\subsection{Equilibrium Shapes}

We next use the Cahn-Hoffman $\xi$-vector formalism [22,14] to determine the equilibrium shapes given by the anisotropy of the interfacial energy. The $\xi$-vector is defined by

$$
\vec{\xi}=\gamma \vec{r}+\gamma_{\phi} \vec{\phi}+\frac{\gamma_{\theta}}{\sin \phi} \vec{\theta}=\nabla[r \gamma(\phi, \theta)],
$$

where the radial unit vector $\vec{r}(\theta, \phi)=\vec{n}$ is normal to the interface. The unit vectors $\vec{\theta}$ and $\vec{\phi}$ are tangent to the interface. The $\xi$-vector reduces to the form $\vec{\xi}=\gamma \vec{n}$ for the isotropic case (constant $\gamma$ ). In the anisotropic case, the $\xi$-vector is in the direction of the normal to the $1 / \gamma(\theta, \phi)$ plot defined by $r=1 / \gamma(\theta, \phi)$. Its component in the radial direction is $\gamma$. To obtain the equilibrium shape, the "tail" of the $\xi$-vector is translated to the origin for each orientation; the collection of all the $\vec{\xi}$-vectors is then the $\vec{\xi}$-surface. The $\vec{\xi}$-surface minimizes the surface energy for a fixed volume if it is a convex shape; in that case it is identical to the Wulff shape [25, 40]. In other cases, the $\vec{\xi}$-surface is not convex and intersects itself, exhibiting "ears" (see, e.g., [7]). If the ears are excluded from the $\vec{\xi}$-surface, the remaining surface represents the equilibrium Wulff shape.

In order to compute the $\vec{\xi}$-surface for A1-L1 $1_{0}$ IPBs, $\gamma$ is first obtained in terms of $\phi$ numerically on a regularly spaced mesh with $\Delta \phi=1.5^{\circ}$; our solutions are independent of $\theta$. By using the

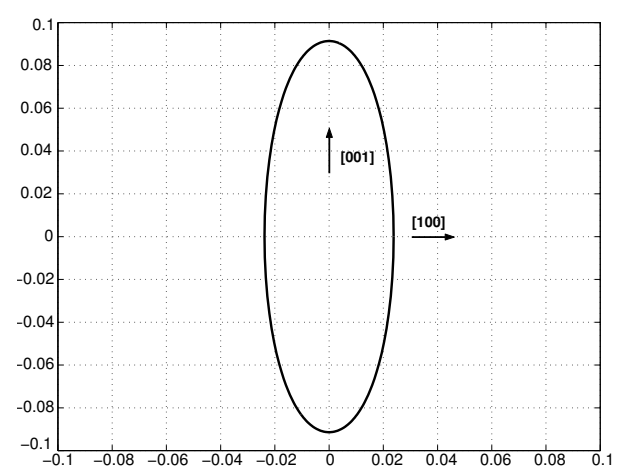

FIG. 11. A cross section of the equilibrium shape for $\mathrm{A} 1-\mathrm{L} 1_{0}$ IPBs. Here $T=2.5, \delta^{2}=1.0$, and $\epsilon^{2}=0.05$. 
formulas given in the appendix of [51], we compute $\vec{\xi}$-surface data in Cartesian coordinates. For those explicit formulas, we employ centered finite difference formulas using the discrete data in order to approximate $\gamma_{\phi}$.

A cross section of the equilibrium shape for the transversely isotropic A1-L1 $1_{0}$ IPBs $(\epsilon \neq 0)$ is shown in Fig. 11. The equilibrium shape is a body of revolution about [001]; the cross section perpendicular to that axis is thus a circle. The long side regions include the low-energy orientations at and near $\langle h k 0\rangle ;[001]$ and nearby orientations have high energy and contribute little to the area of the equilibrium shape. A consequence of the increase in the level of anisotropy as $\epsilon \rightarrow 0$ is that the equilibrium shapes are increasingly elongated; they do not appear to develop missing orientations for any finite value of $\epsilon$, however.

\section{Summary and discussion}

We present a model for the free energy of a binary alloy which incorporates a model phase diagram and can be used to compute properties of diffuse-interfaces; the model uses one conserved order parameter (the composition), and three non-conserved order parameters [8]. The model can describe a variety of phase diagrams; a series of diagrams topologically similar to the $\mathrm{Au}-\mathrm{Cu}$ system was presented in [8]. It can be used for other ordered crystal structures that occur in fcc and other systems [13], though more order parameters may be required. This method allows the computation of interfaces for all orientations at a wide variety of conditions. Other methods, notably CVM, can approximate quite complicated phase diagrams, but are difficult to implement except for interfaces with low-index directions. The method is a powerful tool for computing interfaces and their motion in microstructure evolution with a natural way of incorporating anisotropy [6], and using realistic phase diagrams.

For a phase diagram containing the $\mathrm{A} 1$ phase and the ordered $\mathrm{L} 1_{0}$ and $\mathrm{L} 1_{2}$ phases, similar to what is seen in the $\mathrm{Au}-\mathrm{Cu}$ system, we present a catalog of the interfaces. There are IPBs that can coexist between the disordered $\mathrm{A} 1$ phase and the ordered $\mathrm{L} 1_{0}$ and $\mathrm{L} 1_{2}$ phases, and between the two ordered phases. The symmetry of the underlying lattice can be used to deduce that only one variant of each ordered phase is necessary to compute the anisotropy of the interfacial energy for the orderdisorder IPBs; all other cases are related by simple rotation to these cases. The order-order IPBs require that the two variants of the $\mathrm{L} 1_{0}$ phase be used with a single $\mathrm{L} 1_{2}$ variant in order to compute the possible interfacial energy anisotropies; all other cases can be found by simple rotations of these two results. There are also APBs in the single phase regions of the ordered phases. For the $\mathrm{L}_{2}$ phase, only one case must be computed; for the $\mathrm{L} 1_{0}$ phase, two cases must be computed. Our model can compute all seven of these cases using a single formulation spanning the whole phase diagram.

The free energy density employed in this work and in [48] allows a generalization of previous work on $\mathrm{A} 1$ and $\mathrm{L}_{2}$ in which the composition was held fixed at the stoichiometric value for the $\mathrm{L1}_{2}$ phase throughout the interface [5, 7, 6]. That free energy also prevented the consideration of the $\mathrm{A} 1-\mathrm{L} 1_{0}$ IPB due to the absence of coexistence between the $\mathrm{A} 1$ and $\mathrm{L} 1_{0}$ phases [41]. The new free energy allows us to focus on the interface profile and anisotropy for A1-L1 0 IPBs; equilibrium shapes are also computed by considering only the interfacial energy (elastic effects and lattice mismatch are neglected).

Mathematically, the problem for planar A1-L1 $1_{0}$ IPBs is a non-linear boundary value problem which has a solution that connects two different states in the dependent variables (a heteroclinic connection). From our numerically computed solutions, we observe the profile of the interfaces, as 
well as compute interfacial energies. Once the energies are known, we compute equilibrium shapes using the $\xi$-vector.

Although we find that the IPBs energies are transversely isotropic, we also found strong anisotropy with variations in the polar angle $\phi$. The $\phi$ orientation dependence only served to widen or narrow the interfaces, and no wetting behavior by other phases was observed. The computed compositions always varied smoothly and monotonically between the value for the A1 phase, low in this half of the phase diagram, to the higher value in the $\mathrm{L} 1_{0}$ phase. At the $\mathrm{A} 1-\mathrm{L} 1_{0}$ congruent point the composition was constant throughout the IPB; this necessarily occurred due to the symmetry of our chosen free energy and resulting phase diagram; it is not expected in a real system. Anisotropy of interfaces has also been studied using the CVM (for example [30,36]); to our knowledge, the CVM has not been used to study the $\mathrm{A} 1-\mathrm{L} 1_{0}$ interface.

The transversely isotropic surface energy that we have found highlights an unexpected difficulty in using symmetry arguments to predict the surface energy anisotropy of diffuse interfaces from the high symmetry of the gradient energy. Orientation dependent surface energies in crystalline systems, and the associated Wulff shapes, reflect the symmetries of the crystals, but they are not tensor properties. Gradient energy coefficients can sometimes be shown to transform like tensors, and then the orientation dependence of $\gamma$ can be deduced from the symmetry properties of such tensors. Tensors of rank 2 are transversely isotropic to three-fold, four-fold, and six-fold axes, and thus isotropic for cubic symmetry [43]. Tensors of rank 4 are transversely isotropic to three-fold and six-fold axes. Tensors of rank 4 for a cubic system are described by three numbers and are anisotropic, unless there is a special relationship among these numbers. With an energy that is quadratic in the gradient of a single dependent variable, the gradient energy coefficient is a tensor of rank 2. We showed that the coefficients of an energy quadratic in the gradients of the three dependent variables, $X, Y$ and $Z$, do not behave as tensors. Nonetheless for the fcc system we found them to be described by two of the three non-zero elements that appear in a tensor of rank 4 with cubic symmetry. This is consistent with what we found for the A1-L1 IPB; the Wulff shape has a marked anisotropy consistent with the cubic symmetry. But we now have found two surprises: firstly, transverse isotropy in $\gamma$ and in the Wulff shape for the A1-L $1_{0}$ IPB with only tetragonal symmetry is greater symmetry than expected. Secondly, we found less symmetry in the hcp investigation; there was no isotropy transverse to the six-fold axis [13]. Here the gradient energy coefficient bore no resemblance to elements in a tensor of rank 4 , which not only confirms that these coefficients are not tensors, but forms a strong counterexample to any conjecture that the orientation dependence of $\gamma$ of a diffuse interface might reflect that of a low rank tensor. Both the fcc and the hcp systems each feature three non-conserved order parameters $X_{j}$ and a conserved order parameter $W$ and the free energy functional for each model has the general form

$$
\mathcal{F}=\int_{V}\left\{c_{i j k l} X_{i, j} X_{k, l}+c_{0}|\nabla W|^{2}+f\left(X_{1}, X_{2}, X_{3}, W, T\right)\right\} \mathrm{d} V .
$$

The three non-conserved order parameters $X_{j}$ and the gradient energy coefficients $c_{i j k l}$ do not transform as tensors under changes of coordinates. For the hcp crystal, the disordered state where either type of atom is equally likely to occur on any of the four sublattices, the structure is designated A3 in Strukturbericht notation. When one of the sublattices is occupied (on average) by a different atom than the other three, the crystal structure is denoted $\mathrm{DO}_{19}$; this ordered state is exactly analogous to the $\mathrm{L}_{2}$ structure on an fcc lattice [13].

If one were to reason by analogy with the elastic case, then both the transverse isotropy of the $\mathrm{A} 1-\mathrm{L} 1_{0}$ IPB surface energy and the six-fold anisotropy of the A3-DO ${ }_{19}$ IPB surface energy 
is unexpected. We also note that the $\mathrm{A} 1-\mathrm{L} 1_{0}$ IPB involves a single non-zero non-conserved order parameter, whereas the $\mathrm{A} 1-\mathrm{L}_{2}$ and $\mathrm{A} 3-\mathrm{DO}_{19}$ IPB involve three non-zero non-conserved order parameters. In addition, wetting by a third phase also occurs for the latter two cases, which plays a role in the observed transverse anisotropy for these IPBs. If the three non-conserved order parameters are constrained to remain equal throughout the interfacial region (which precludes wetting), the resulting system is effectively a single-order-parameter model, and an isotropic surface energy results.

The $\mathrm{A} 1-\mathrm{L} 1_{0}$ equilibrium shapes resulting from $\xi$-vector calculations are bodies of revolution, and no missing orientations occurred for the parameters we studied. The cross section of the anisotropic equilibrium shape (in the absence of elastic effects) is roughly an ellipse for fixed azimuthal angle $\theta$ and exactly a circle for fixed polar angle $\phi$ relative to the $c$-axis of the tetragonal $\mathrm{L}_{0}$ ([001] for the case we considered). This is in contrast to the cuboidal, nearly spherical equilibrium Wulff shapes found in our previous work for $\mathrm{A} 1-\mathrm{L} 1_{2}$ IPBs [5, 7] on an fcc lattice; that $\gamma$ had six equal minima in the cube directions. Another contrast may be found with A3-DO 19 IPBs in an hep binary alloy [13]; in that situation, there is six-fold anisotropy in the plane orthogonal to the hexagonal axis. Nearly spherical equilibrium shapes are found as well but with six minima in $\gamma$ distributed evenly around the equator. A concentration variable must be added to the hcp model of [13] and a phase diagram generated, in order to consider the analogous cases studied in this paper.

The variable composition added the capability to satisfy the Gibbs adsorption equation. We have verified that our model now satisfies the Gibbs adsorption equation and provides an instance of the diffuse interface generalization of sharp interface results, as discussed in [11]. We have also verified that the numerical results obtained from the computed IPBs are in agreement with this identity.

A variation in the width of the interface with orientation is noticeable, but it does not show a dramatic widening as in cases when wetting of the phase boundary occurs (e.g., as in the A1-L12 case [7, 48]). However, in the case when $\epsilon$ is very small and one is at the congruent point, one must be able to resolve very thin $\langle h k 0\rangle$ interfaces (compared to [001]). In such a computation with finite difference or finite element discretizations, an adaptive mesh approach should be effective. For fixed mesh approaches, the mesh (or for a spectral method, the number of modes) must be able to resolve the thinnest interfaces.

\section{Appendix A}

The non-dimensional form of the Helmholtz free energy is given in the following

$$
\begin{aligned}
F(X, Y, Z, W, T)= & e_{0}(W)+e_{2}(W)\left(X^{2}+Y^{2}+Z^{2}\right)+e_{3}(W) X Y Z \\
& +e_{41}\left(X^{4}+Y^{4}+Z^{4}\right)+e_{42}\left(X^{2} Y^{2}+X^{2} Z^{2}+Y^{2} Z^{2}\right)+\frac{T}{4} \sum_{j=1}^{4} I\left(\rho_{j}\right),
\end{aligned}
$$

where $I(x)$ is given in (4).

The right sides of the equations [15] are given as follows:

$$
\begin{aligned}
F_{Z}(X, Y, Z, W, T)= & 2 e_{2} Z+e_{3} X Y+4 e_{41} Z^{3}+2 e_{42} Z\left(X^{2}+Y^{2}\right) \\
& +\frac{T}{4} \ln [Q(X, Y, Z, W)], \\
F_{W}(X, Y, Z, W, T)= & e_{0, W}+e_{2, W}\left(X^{2}+Y^{2}+Z^{2}\right)+e_{3, W} X Y Z \\
& +\frac{T}{4} \ln [R(X, Y, Z, W)] .
\end{aligned}
$$


$Q$ and $R$ are defined as follows:

$$
\begin{aligned}
Q(X, Y, Z, W)= & \frac{(W+X+Y+Z)(W-X-Y+Z)}{(1-W-X-Y-Z)(1-W+X+Y-Z)} \\
& \times \frac{(1-W-X+Y+Z)(1-W+X-Y+Z)}{(W+X-Y-Z)(W-X+Y-Z)},
\end{aligned}
$$

and

$$
\begin{aligned}
R(X, Y, Z, W)= & \frac{(W+X+Y+Z)(W+X-Y-Z)}{(1-W-X-Y-Z)(1-W-X+Y+Z)} \\
& \times \frac{(W-X+Y-Z)(W-X-Y+Z)}{(1-W+X-Y+Z)(1-W+X+Y-Z)} .
\end{aligned}
$$

These expressions are used with $X=Y=0$ in this paper and with $Z=0$ where appropriate.

\section{Appendix B}

Here we provide a short derivation of the Gibbs adsorption equation for our model of the A1-L10 IPB with a non-zero order parameter $Z=Z(\zeta)$, overall composition $W=W(\zeta)$, and free energy density $F_{\mathrm{L}_{1}}(Z, W, T)$; a fuller discussion in the context of general diffuse interface theories is given in [37].

For the free energy functional

$$
\mathcal{F}=\int_{\infty}^{\infty}\left\{\frac{\epsilon^{2}}{2} Z_{\zeta}^{2}+\frac{\delta^{2}}{2} W_{\zeta}^{2}+F_{\mathrm{L}_{0}}(Z, W, T)\right\} \mathrm{d} \zeta,
$$

the Euler equations, $\delta \mathcal{F} / \delta W=\mu$ and $\delta \mathcal{F} / \delta Z=0$, yield

$$
\begin{aligned}
F_{\mathrm{L} 1_{0}, W}(Z, W, T) & =\delta W_{\zeta \zeta}+\mu, \\
F_{\mathrm{L1}_{0}, Z}(Z, W, T) & =\epsilon^{2} Z_{\zeta \zeta},
\end{aligned}
$$

and admit a first integral given by

$$
\frac{\epsilon^{2}}{2} Z_{\zeta}^{2}+\frac{\delta^{2}}{2} W_{\zeta}^{2}=F_{\mathrm{L} 1_{0}}(Z, W, T)-F_{\mathrm{L} 1_{0}}\left(0, W_{\mathrm{A} 1}, T\right)-\left(W-W_{\mathrm{A} 1}\right) \mu \equiv \Delta F .
$$

Here

$$
W \rightarrow W_{\mathrm{A} 1}, \quad Z \rightarrow 0, \quad \text { as } \zeta \rightarrow-\infty
$$

and

$$
W \rightarrow W_{\mathrm{L} 1_{0}}, \quad Z \rightarrow Z_{\mathrm{L} 1_{0}}, \quad \text { as } \zeta \rightarrow \infty,
$$

give the far-field values of $W$ and $Z$.

Evaluating the Euler equations and first integral in the far fields gives the common tangent conditions (8) that relate $W_{\mathrm{A} 1}, W_{\mathrm{L}_{0}}, Z_{\mathrm{L}_{0}}$, and $T$,

$$
\begin{aligned}
\mu & =F_{\mathrm{L} 1_{0}, W}\left(0, W_{\mathrm{A} 1}, T\right)=F_{\mathrm{L} 1_{0}, W}\left(Z_{\mathrm{L} 1_{0}}, W_{\mathrm{L} 1_{0}}, T\right) \\
& =\frac{\left[F_{\mathrm{L}_{0}}\left(Z_{\mathrm{L}_{0}}, W_{\mathrm{L} 1_{0}}, T\right)-F_{\mathrm{L} 1_{0}}\left(0, W_{\mathrm{A} 1}, T\right)\right]}{\left(W_{\mathrm{L} 1_{0}}-W_{\mathrm{A} 1}\right)} .
\end{aligned}
$$


The common tangent conditions, and the equation $F_{\mathrm{L} 1_{0}, Z}\left(Z_{\mathrm{L}_{0}}, W_{\mathrm{L} 1_{0}}, T\right)=0$, provide three relations between the four parameters $W_{\mathrm{A} 1}, W_{\mathrm{L} 1_{0}}, Z_{\mathrm{L} 1_{0}}$, and $T$, consistent with the single degree of freedom for coexistence of the bulk phases along the phase diagram. We choose to regard the temperature as the degree of freedom, and write $W_{\mathrm{A} 1}=W_{\mathrm{A} 1}(T)$ and $W_{\mathrm{L} 1_{0}}=W_{\mathrm{L} 1_{0}}(T)$.

The surface energy is given by

$$
\gamma=\int_{-\infty}^{\infty}\left\{\frac{\epsilon^{2}}{2} Z_{\zeta}^{2}+\frac{\delta^{2}}{2} W_{\zeta}^{2}+\Delta F\right\} \mathrm{d} \zeta
$$

Since the solution can be considered to be a function of the prescribed temperature $T$, with $W=$ $W(\zeta ; T)$ and $Z=Z(\zeta ; T)$, the surface energy of the IPB is a function of $T$ as well. We then have

$$
\frac{\mathrm{d} \gamma}{\mathrm{d} T}=\int_{-\infty}^{\infty}\left\{\epsilon^{2} Z_{\zeta} Z_{\zeta T}+\delta^{2} W_{\zeta} W_{\zeta T}+\frac{\mathrm{d} \Delta F}{\mathrm{~d} T}\right\} \mathrm{d} \zeta,
$$

which can be integrated by parts to obtain

$$
\frac{\mathrm{d} \gamma}{\mathrm{d} T}=\int_{-\infty}^{\infty}\left\{-\epsilon^{2} Z_{\zeta \zeta} Z_{T}-\delta^{2} W_{\zeta \zeta} W_{T}+\frac{\mathrm{d} \Delta F}{\mathrm{~d} T}\right\} \mathrm{d} \zeta .
$$

We have

$$
\begin{aligned}
\frac{\mathrm{d} \Delta F}{\mathrm{~d} T}= & F_{\mathrm{L} 1_{0}, W}(Z, W, T) W_{T}+F_{\mathrm{L} 1_{0}, Z}(Z, W, T) Z_{T}+F_{\mathrm{L}_{0}, T}(Z, W, T) \\
& -F_{\mathrm{L}_{0}, W}\left(0, W_{\mathrm{A} 1}, T\right) \frac{\mathrm{d} W_{\mathrm{A} 1}}{\mathrm{~d} T} \\
& -F_{\mathrm{L}_{0}, T}\left(0, W_{\mathrm{A} 1}, T\right)-F_{\mathrm{L}_{0}, W}\left(0, W_{\mathrm{A} 1}, T\right) W_{T}+F_{\mathrm{L}_{0}, W}\left(0, W_{\mathrm{A} 1}, T\right) \frac{\mathrm{d} W_{\mathrm{A} 1}}{\mathrm{~d} T} \\
& -\left(W-W_{\mathrm{A} 1}\right) F_{\mathrm{L}_{0}, W W}\left(0, W_{\mathrm{A} 1}, T\right) \frac{\mathrm{d} W_{\mathrm{A} 1}}{\mathrm{~d} T}-\left(W-W_{\mathrm{A} 1}\right) F_{\mathrm{L} 1_{0}, W T}\left(0, W_{\mathrm{A} 1}, T\right) .
\end{aligned}
$$

The bulk chemical potential is given by

$$
\mu(T)=F_{\mathrm{L} 1_{0}, W}\left(0, W_{\mathrm{A} 1}(T), T\right),
$$

and its temperature derivative is

$$
\frac{\mathrm{d} \mu}{\mathrm{d} T}=F_{\mathrm{L} 1_{0}, W W}\left(0, W_{\mathrm{A} 1}, T\right) \frac{\mathrm{d} W_{\mathrm{A} 1}}{\mathrm{~d} T}+F_{\mathrm{L} 1_{0}, W T}\left(0, W_{\mathrm{A} 1}, T\right) .
$$

The entropy is $S=-F_{\mathrm{L} 1_{0}, T}(Z, W, T)$, with

$$
S_{\mathrm{A} 1}=-F_{\mathrm{L} 1_{0}, T}\left(0, W_{\mathrm{A} 1}, T\right), \quad S_{\mathrm{L} 1_{0}}=-F_{\mathrm{L} 1_{0}, T}\left(Z_{\mathrm{L} 1_{0}}, W_{\mathrm{L} 1_{0}}, T\right) .
$$

If we use these definitions, the expression for $\mathrm{d} \Delta F / \mathrm{d} T$ simplifies to give

$$
\begin{aligned}
\frac{\mathrm{d} \Delta F}{\mathrm{~d} T}= & {\left[F_{\mathrm{L} 1_{0}, W}(Z, W, T)-F_{\mathrm{L}_{0}, W}\left(0, W_{\mathrm{A} 1}, T\right)\right] W_{T}+F_{\mathrm{L} 1_{0}, Z}(Z, W, T) Z_{T} } \\
& -\left[S-S_{\mathrm{A} 1}\right]-\left(W-W_{\mathrm{A} 1}\right) \frac{\mathrm{d} \mu}{\mathrm{d} T} .
\end{aligned}
$$


Inserting this expression into (42) gives

$$
\begin{array}{r}
\frac{\mathrm{d} \gamma}{\mathrm{d} T}=\int_{-\infty}^{\infty}\left\{\left[F_{\mathrm{L}_{0}, Z}(Z, W, T)-\epsilon^{2} Z_{\zeta \zeta}\right] Z_{T}+\left[F_{\mathrm{L}_{0}, W}(Z, W, T)-F_{\mathrm{L}_{0}, W}\left(0, W_{\mathrm{A} 1}, T\right)-\delta W_{\zeta \zeta}\right] W_{T}\right. \\
\left.-\left[S-S_{\mathrm{A} 1}\right]-\left(W-W_{\mathrm{A} 1}\right) \frac{\mathrm{d} \mu}{\mathrm{d} T}\right\} \mathrm{d} \zeta
\end{array}
$$

The first two terms vanish by virtue of the Euler equations, leading to

$$
\frac{\mathrm{d} \gamma}{\mathrm{d} T}=-\int_{-\infty}^{\infty}\left\{\left[S-S_{\mathrm{A} 1}\right]+\left(W-W_{\mathrm{A} 1}\right) \frac{\mathrm{d} \mu}{\mathrm{d} T}\right\} \mathrm{d} \zeta .
$$

Here we note that $\mathrm{d} \mu / \mathrm{d} T$ is independent of $\zeta$. It is easily seen that

$$
\left(W_{\mathrm{L}_{0}}-W_{\mathrm{A} 1}\right) \frac{\mathrm{d} \mu}{\mathrm{d} T}=-\left(S_{\mathrm{L}_{0}}-S_{\mathrm{A} 1}\right),
$$

which can be used to provide the alternative form

$$
\frac{\mathrm{d} \gamma}{\mathrm{d} T}=-\int_{-\infty}^{\infty}\left\{\left(S-S_{\mathrm{A} 1}\right)-\left(W-W_{\mathrm{A} 1}\right) \frac{S_{\mathrm{L} 1_{0}}-S_{\mathrm{A} 1}}{W_{\mathrm{L} 1_{0}}-W_{\mathrm{A} 1}}\right\} \mathrm{d} \zeta
$$

\section{Acknowledgments}

GBM would like to thank the National Aeronautics and Space Administration Microgravity Research Program for support. RJB would like to thank the NSF for support via grants DMS9722854 and DMS-9631287. GBT would like to thank the Turkish Ministry of Education for support. The authors are grateful for helpful discussions with A. A. Wheeler.

\section{REFERENCES}

1. Ahmad, A., Wheeler, A. A., Boettinger, W. J., \& McFadden, G. B. Solute trapping and solute drag in a phase-field model of rapid solidification. Phys. Rev. E 58 (1998), 3436-3450.

2. Allen, S. M. \& CAHN, J. W. A microscopic theory for antiphase boundary motion and its application to antiphase domain coarsening. Acta Metall. Mater. 27 (1979), 1085-1095.

3. Asher, U., Christiansen, J., \& Russell, R. D. Collocation software for boundary-value ODEs. ACM Trans. Math. Software 7 (1981), 209-222. Zbl 0455.65067

4. Boettinger, W. J. \& WARren, J. A. Simulation of the cell to plane front transition during directional solidification at high velocity. J. Crystal Growth 200 (1999), 583.

5. Braun, R. J., Cahn, J. W., Hagedorn, J., McFadden, G. B., \& Wheeler, A. A. Anisotropic interfaces and ordering in fcc alloys: A multiple-order-parameter continuum theory. Mathematics of Microstructure Evolution, L.-Q. Chen et al. (eds.), TMS/SIAM, Philadelphia, PA, 1996, 225-244.

6. Braun, R. J., Cahn, J. W., McFadden, G. B., Rushmeier, H. E., \& Wheeler, A. A. Theory of anisotropic growth rates in the ordering of an F.C.C. alloy. Acta Mater. 46 (1997), 1-12.

7. Braun, R. J., Cahn, J. W., McFadden, G. B., \& Wheeler, A. A. Anisotropy of interfaces in an ordered alloy: a multiple-order-parameter model. Trans. Roy. Soc. London A 355 (1997), 1787-1833. Zbl pre01089140

8. Braun, R. J., Zhang, J., Cahn, J. W., McFadden, G. B., \& Wheeler, A. A. Model phase diagrams for an fcc alloy. Interfaces for the Twenty-First Century, M. K. Smith et al. (eds.), Imperial College Press, London, 2002, 213-230. 
9. Caginalp, G. \& XIE, W. Phase-field and and sharp-interface alloy models. Phys. Rev. E 48 (1993), 1897-1909. MR 96m:82022

10. CAhn, J. W. On spinodal decomposition. Acta Metall. 9 (1961), 795-801.

11. CAHN, J. W. Thermodynamics of solid and fluid interfaces. Interfacial Segregation, ASM, Metals Park, OH, 1977, 3-23.

12. CAhn, J. W. \& Allen, S. M. A microscopic theory for domain wall motion and its experimental verification in Fe-Al alloy domain growth kinetics. J. Phys. (Paris) Colloque 38 (1977) C7 51-54.

13. Cahn, J. W., Han, S. C., \& MCFAdden, G. B. Anisotropy of interfaces in an ordered HCP binary alloy. J. Statist. Phys. 95 (1999), 1337-1360. Zbl pre01366092 MR 1712450

14. Cahn, J. W. \& Hoffman, D. W. A vector thermodynamics for anisotropic surfaces. II. Curved and faceted surfaces. Acta Metall. 22 (1977), 1205-1214.

15. CAHN, J. W. \& KIKUCHI, R. Theory of domain walls in ordered structures-III. Effect of substitutional deviations from stoichiometry. J. Phys. Chem. Solids 27 (1966), 1305-1317.

16. Danan, F., Chen, L.-Q., Chen, S. P., \& Voorhees, P. W. Phase field formulations for modeling the Ostwald ripening in two phase systems. Comp. Materials Sci. 9 (1998), 329-336.

17. Ducastelle, F. Order and Phase Stability in Alloys, North-Holland, New York, 1991.

18. FAN, D. \& CHEN, L. Q. Microstructural evolution and grain growth kinetics in a two-phase solid with quadrijunctions. Mathematics of Microstructure Evolution, L.-Q. Chen et al. (eds.), TMS/SIAM, Philadelphia, PA, 1996, 215-223.

19. FInEL, A. Thermodynamical properties of antiphases in FCC ordered alloys. Ordering and Disordering in Alloys, A. Yavari (ed.), Elsevier Appl. Sci., New York, 1992, 182-193.

20. De Fontaine, D. Cluster approach to order-disorder transformations in alloys. Solid State Phys. 47 (1994), 33-176.

21. Hilliard, J. E. Spinodal decomposition. Phase Transformations, H. I. Aaronson (ed.), Amer. Soc. Metals, Metals Park, OH, 1970, 497-560.

22. Hoffman, D. W. \& CAhN, J. W. A vector thermodynamics for anisotropic surfaces. I. Fundamentals and application to plane surface junctions. Surface Sci. 31 (1972), 368-388.

23. JAMES, R. D. \& HANE, K. F. Martensitic transformations and shape-memory materials. Acta Mater. 48 (2000), 197-222.

24. Johnson, W. C. \& CAhn, J. W. Elastically induced shape bifurcations of inclusions. Acta Mater. 32 (1984), 1925-1933.

25. Johnson, C. A. \& Chakerian, G. D. On the proof and uniqueness of Wulff's construction of the shape of minimum surface free energy. J. Math. Phys. 6 (1965), 1403-1404. Zbl 0149.19103 MR 31 \#5628

26. Johnson, W. C. \& VoorheEs, P. W. Elastic interaction and stability of misfitting cuboidal inhomogeneities. J. Appl. Phys. 61 (1987), 1610-1619.

27. Johnson, W. C. \& Voorhees, P. W. Elastically-induced precipitate shape transitions in coherent solids. Solid State Phenom. 23 (1992), 87-104.

28. Khachaturyan, A. G. Theory of Structural Transformations in Solids. Wiley, New York, 1983.

29. KIKUCHI, R. Solution of the controversy in the fcc-based phase diagram. Progr. Theoret. Phys. Suppl. 87 (1986), 69-76.

30. KikUChi, R. \& CAHn, J. W. Theory of interphase and antiphase boundaries in FCC alloys. Acta Metall. 27 (1979), 1337-1353.

31. KikUChi, R. \& DE Fontaine, D. Theoretical calculations of phase diagrams using the cluster variation method. Application of Phase Diagrams in Metallurgy and Ceramics, C. G. Carter (ed.), National Bureau of Standards Special Publication 496, 1978, 967-998.

32. Kikuchi, R., Sanchez, J. M., De Fontaine, D., \& Yamauchi, H. Theoretical calculation of the $\mathrm{Cu}-\mathrm{Ag}-\mathrm{Au}$ coherent phase diagram. Acta Metall. 28 (1980), 651-662. 
33. Kobayashi, R. Modeling and numerical simulations of dendritic crystal growth. Phys. D 63 (1993), 410-423. Zbl 0797.35175

34. LaI, Z.-W. Theory of ordering dynamics for $\mathrm{Cu}_{3}$ Au. Phys. Rev. B 41 (1990), 9239-9256.

35. Lifshitz, E. M. \& PitaevskiI, L. P. Statistical Physics, Part 1. 3rd ed., Pergamon, Oxford, 1980, 459-471.

36. Mazauric, V. G. Phase diagram of domain walls in the cubic superstructures of the fcc lattice. J. Comput.-Aided Mater. Des. 4 (1997), 113-132.

37. McFadden, G. B. \& WheEler, A. A. On the Gibbs adsorption equation for diffuse interface models. Proc. Roy. Soc. London Ser. A Math. 458 (2002), 1129-1149. Zbl 0996.82023 MR 2003b:82043

38. McFadden, G. B., Wheeler, A. A., Braun, R. J., Coriell, S. R., \& Sekerka, R. F. Phase-field models for anisotropic interfaces. Phys. Rev. E 48 (1993), 2016-2024. MR 1377920

39. Muddle, B. C., Nie, J. F., \& Hugo, G. R. Application of the theory of martensite crystallography to displacive phase transformations in substitutional nonferrous alloys. Metal. and Mat. Trans. A 25 (1994), 1841-1856.

40. Mullins, W. W. Proof that the two-dimensional shape of minimum surface free energy is convex. J. Math. Phys. 3 (1962), 754-759. Zbl 0112.23801

41. NiX, F. C. \& Shockley, W. Order-disorder transformations in alloys. Rev. Modern Phys. 10 (1938), $1-71$.

42. Novick-CoHen, A., \& CAHN, J. W. Evolution equations for phase separation and ordering in binary alloys. J. Statist. Phys. 76 (1994), 877-909. Zbl 0840.35110

43. Nye, J. F. Physical Properties of Crystals. Oxford Univ. Press, Oxford, 1957. Zbl 0079.22601

44. Okamoto, H., Chakrabarti, D. J., Laughlin, D. E., \& Massalski, T. B. The Au-Cu (GoldCopper) system. Bull. Alloy Phase Diagrams 8 (1987), 454-474.

45. Powell, M. J. D. A hybrid method for nonlinear equations. Numerical Methods for Nonlinear Algebraic Equations, P. Rabinowitz (ed.), Gordon and Breach, New York, 1988, 87-161. Z Zbl 0277.65028 MR 49 \#8330a

46. RAYLEIGH, LORD On the theory of surface forces. II. Compressible fluids. Philos. Mag. 33 (1892), 209220.

47. Slatec Common Math Library, National Energy Software Center, Argonne National Laboratory, Argonne, IL. The program SNSQ was written by K. L. Hiebert and is based on an algorithm of M. J. D. Powell.

48. TANoĞLU, G. B. Phase boundaries and anisotropy via multiple-order-parameter theory for an fcc alloy. Ph.D. thesis, Univ. of Delaware, 2000.

49. TANoĞLU, G. B. \& BRAun, R. J. Anisotropy of $A 1-L 1_{2}$ and $L 1_{0}-L 1_{2}$ interphase boundaries via multiple-order-parameter theory. In preparation.

50. TAYlor, J. E. \& CAhn, J. W. Diffuse interfaces with sharp corners and facets: Phase field modeling of strongly anisotropic surfaces. Phys. D 112 (1998), 381-411. Zbl 0930.35201 MR 98i:35185

51. Voorhees, P. W., Coriell, S. R., McFadden, G. B., \& Sekerka, R. F. The effect of anisotropic crystal-melt surface tension on grain boundary groove morphology. J. Crystal Growth 67 (1984), 425-440.

52. VAN DER WAALS, J. D. The thermodynamic theory of capillarity under the hypothesis of a continuous variation of density. Verhand. Konink. Akad. Wetensch. Amsterdam (Sect. 1) 1 (1892), 1-56 (in Dutch); English translation: J. S. Rowlinson, J. Statist. Phys. 20 (1979), 197-244.

53. Weinstock, R. Calculus of Variations. Dover Publ., New York, 1972. Zbl 0296.49001 MR 56 \#1856

54. Wheeler, A. A., Boettinger, W. J., \& McFadden, G. B. A phase-field model for isothermal phase transitions in binary alloys, Phys. Rev. A 45 (1992), 7424-7439.

55. Wheeler, A. A., Boettinger, W. J., \& McFadden, G. B. A phase-field model of solute trapping during solidification, Phys. Rev. E 47 (1993), 1893-1909. 